\title{
Perfil do consumo de oxigênio de adolescentes com cardiopatias congênitas.
}

\author{
Satiro Alves Ribeiro Pettinelli ${ }^{1}$ Gabriela Figliolino ${ }^{2}$ Luiz Antônio R. Medina ${ }^{3}$
}

\begin{abstract}
1. Residente em Fisioterapia Cardiorrespiratória pelo Instituto Dante Pazzanese de Cardiologia - IDPC. 2. Fisioterapeuta Especialista em Reabilitação Cardiovascular e Fisioterapia pelo Instituto Dante Pazzanese de Cardiologia - IDPC. Preceptora da Fisioterapia da Universidade Nove de Julho.

3. Fisioterapeuta Especialista em Fisioterapia Cardiorrespiratória e Reabilitação Cardiovascular pelo Instituto Dante Pazzanese de Cardiologia - IDPC. Supervisor da pós-graduação em Fisioterapia Cardiorrespiratória do Instituto Dante Pazzanese de Cardiologia - IDPC.
\end{abstract}

\section{RESUMO}

Introdução: Adolescentes jovens com cardiopatia congênita possuem qualidade de vida com limitações físicas, sociais e emocionais, podendo evoluir com diminuição ou ausência das atividades cotidianas. Para verificar a capacidade funcional é necessário realizar uma avaliação por meio do teste cardiopulmonar e verificar o consumo máximo de oxigênio, limiar anaeróbio, pulso de oxigênio, VE/VCO2 slope, quociente respiratório e consumo máximo de oxigênio para quantificar a gravidade da doença, pois a captação máxima de oxigênio (VO2max) se correlaciona com a qualidade de vida, prognóstico, capacidade funcional, diagnóstico, quantificação de sintomas e avaliação da resposta ao tratamento Objetivo: O objetivo desse estudo foi analisar o perfil do consumo de oxigênio no teste de esforço cardiopulmonar em adolescentes com cardiopatia congênita. Método: Foi realizado um estudo observacional retrospectivo utilizando dados de prontuário de junho de 2018 a dezembro de 2019, com teste cardiopulmonar realizado no Instituto Dante Pazzanese de Cardiologia para traçar o perfil do oxigênio dessa população. Conclusão: Este trabalho evidenciou o perfil de oxigênio dos adolescentes, podendo auxiliar no tratamento conservador, por meio de exercícios de reabilitação cardíaca, para melhorar a qualidade de vida de adolescentes portadores de cardiopatias congênitas.

Palavras-chave: Doença Cardíaca Congênita, Qualidade de Vida, Jovens.

\begin{abstract}
Introduction: Young adolescents with congenital heart disease have quality of life with physical, social and emotional limitations, and may evolue with a decrease or absence of daily activities. To verify the functional capacity, it is necessary to carry out an evaluation through the cardiopulmonary test and verify the maximum oxygen consumption, anaerobic threshold, oxygen pulse, $\mathrm{VE} / \mathrm{VCO} 2$ inclination, quotient respirator and maximum oxygen consumption to quantify a disease lesion, because maximum oxygen uptake (VO2max) correlates with quality of life, prognosis, functional capacity, diagnosis, symptom quantification and assessment of response to treatment. Objective: The objective of this study was to analyze the profile of oxygen consumption without cardiopulmonary stress test in adolescents with congenital heart disease. Method: A retrospective study was performed using medical record data from patients with congenital heart disease, from June 2018 to December 2019, with a cardiopulmonary test
\end{abstract}


performed at the Instituto Dante Pazzanese de Cardiologia. Conclusion: This study showed the oxygen profile of adolescents, which can assist in conservative treatment, through cardiac rehabilitation exercises, to improve the quality of life of adolescents with congenital heart diseases.

Keywords: Congenital heart disease, Quality of life, Youth

\section{INTRODUÇÃO}

A cardiopatia congênita é uma anormalidade estrutural do coração presente antes do nascimento, o qual pode acometer o coração e os grandes vasos. Essas alterações ocorrem enquanto o feto está se desenvolvendo no útero e podem evoluir com hipertensão pulmonar devido ao aumento ou diminuição do fluxo sanguíneo pulmonar onde ocorre alteração do shunt sistêmico-pulmonar ${ }^{1,2}$.

Todas as alterações cardíacas congênitas que apresentem ampla comunicação intra ou extra-cardíaca, principalmente com shunt sistêmico da esquerda para direita, cursam com aumento de pressão na circulação pulmonar e, consequentemente, podem causar Hipertensão Pulmonar, caso o defeito não seja cirurgicamente corrigido na infância ${ }^{3,4}$.

A capacidade funcional de indivíduos com cardiopatia congênita é menor quando comparado com pessoas adultos saudáveis. Para verificar a capacidade funcional é necessário realizar uma avaliação com o teste cardiopulmonar, pois ele associa equipamentos como ergômetros, registradores de eletrocardiograma e dispositivos de medição de pressão sanguínea e das respostas ventilatórias. Além disso ele também pode ser utilizado para determinar a necessidade de intervenções médicas ou cirúrgicas e determinar a eficácia dessas intervenções ${ }^{5,6}$.

Apesar do teste de esforço cardiopulmonar (TECP) ser muito utilizado na prática clínica, ainda existe uma lacuna na literatura a respeito de dados do consumo de oxigênio 
de pacientes adolescentes com cardiopatias congênitas, sendo assim, a relevância de traçar um perfil desses pacientes se deve à necessidade de conhecimento para adequar possíveis condutas clínicas e de reabilitação ${ }^{3,7}$.

A qualidade de vida das pessoas com cardiopatia congênita sofre limitações físicas, sociais e emocionais, podendo evoluir com diminuição ou ausência das atividades cotidianas. O SF-36 é um instrumento genérico de avaliação da qualidade de vida relacionada à saúde, sendo de fácil aplicação e compreensão ${ }^{8}$.

O exercício físico em adolescentes cardiopatas melhora o consumo de oxigênio (VO2 pico), aumenta o débito cardíaco, aumenta a pressão arterial pulmonar e sistêmica e aumenta a sensibilidade a insulina ${ }^{8}$.

Para prescrever o exercício de maneira adequada é necessário verificar: história e exame físico, avaliar cinco parâmetros de linha de base (avaliação da função ventricular, avaliação da pressão arterial pulmonar, avaliação da aorta, avaliação de arritmia e avaliação de saturação em repouso e durante exercício), recomendação do tipo de exercício, TECP, recomendação da intensidade relativa e acompanhamento. A prescrição do exercício pode ser baseada na carga máxima de trabalho. Quando um dos parâmetros está alterado a recomendação é para realizar exercícios moderados ${ }^{8,13}$.

\section{MÉTODOS}

Foi realizada a coleta dos dados do teste de esforço cardiopulmonar em 46 prontuários de adolescentes sedentários diagnosticados com cardiopatia congênita corrigida cirurgicamente, no Instituto Dante Pazzanese de Cardiologia, no período de junho de 2018 a dezembro de 2019, sendo realizada avaliação de: ventilação minuto, 
pressão expirada de oxigênio e dióxido de carbono, quociente respiratório, pulso de oxigênio, VO2 máximo e relação VE/VCO2 slope.

Os valores de referência do consumo de oxigênio máximo obtido pelo teste de esforço cardiopulmonar que foram utilizados são:

Quadro 1: Valores de VO 2 máx relativos à massa corporal $\left(\mathrm{mL}^{\mathrm{k}} \mathrm{kg}^{-1} \cdot \mathrm{min}^{-1}\right)$ e de acordo com a idade

\begin{tabular}{|l|l|l|l|}
\hline \multicolumn{2}{|l|}{ VO2 Máx mL.Kg-1 $\mathbf{m i n}^{-1}$} & Meninas & P \\
\hline & Meninos & $43,53+/-6,65$ & 0,00096 \\
\hline 10 anos & $38,29+/-6,28$ & $42,95+/-6,93$ & 0,0084 \\
\hline 11 anos & $38,58+/-7,10$ & $44,77+/-8,90$ & 0,0001 \\
\hline 12 anos & $37,63+/-5,67$ & $45,49+/-10,41$ & 0,0001 \\
\hline 13 anos & $38,27+/-4,43$ & & $5,8 \times 10^{-13}$ \\
\hline 14 anos & $36,76+/-5,98$ & $49,55+/-7,29$ & \\
\hline
\end{tabular}

Classificação da aptidão cardiorrespiratória pelo consumo máximo de oxigênio medido diretamente pelas faixas etárias de 10 a 14 anos.

Quadro 2: Valores do VO2 máx de acordo o gênero distribuído por quintis. Proposta de classificação para a faixa etária de 10 a 14 anos em cinco categorias

\begin{tabular}{|l|l|l|}
\hline & Meninas & Meninos \\
\hline Muito fraca & $<33,0$ & $<38,7$ \\
\hline Fraca & $33,0-36,4$ & $38,7-43,3$ \\
\hline
\end{tabular}




\begin{tabular}{|l|l|l|}
\hline Regular & $36,5-38,7$ & $43,4-47,9$ \\
\hline Boa & $38,8-42,4$ & $48,0-52,2$ \\
\hline Excelente & $>/ 42,5$ & $>/ 52,3$ \\
\hline
\end{tabular}

\section{Desenho do Estudo}

Estudo observacional retrospectivo com dados de prontuário de junho de 2018 a dezembro de 2019, com teste cardiopulmonar realizado no Instituto Dante Pazzanese de Cardiologia nesse período.

\section{Elegibilidade}

\section{Critérios de inclusão}

- Ambos os sexos;

- Idade entre 15 e 22 anos;

- Teste cardiopulmonar realizado entre janeiro de 2010 e janeiro de 2019;

- Termo de compromisso livre e esclarecido assinado pelos responsáveis;

- Termo de assentimento (TALE);

- Diagnóstico de cardiopatia congênita.

\section{Critérios de exclusão}

- Prontuários com dados faltantes;

- Pacientes que necessitam de uso contínuo de oxigênio.

\section{Fluxograma}


Foram consultados os dados obtidos por análise de prontuário. Após a obtenção dos dados do teste cardiopulmonar foi verificado o consumo de oxigênio e analisada classificação.

\section{Avaliação}

Foi realizada uma avaliação dos participantes antes do estudo por meio da utilização de um instrumento que continha a caracterização da amostra e dados do teste de esforço cardiopulmonar.

\section{- Caracterização da amostra}

A caracterização da amostra foi composta por um instrumento simples de avaliação que conteve informações como sexo, peso, idade, altura, telefone para contato, história da doença atual, antecedentes pessoais, hipótese diagnóstica, resultados de ecocardiograma, cateterismo cardíaco, medicamentos em uso e classificação funcional da New York Heart Association.

\section{- Teste de esforço cardiopulmonar (TECP)}

O TECP fornece informações de respiração por respiração: captação pulmonar de oxigênio $\left(\dot{\mathrm{V}}_{2}, \mathrm{ml} / \mathrm{min}\right)$, eliminação de dióxido de carbono $\left(\dot{\mathrm{VCO}}_{2}, \mathrm{ml} / \mathrm{min}\right)$, razão de trocas respiratórias $(\mathrm{R})$, ventilação minuto $\left(\mathrm{V}_{\mathrm{E}}, 1 / \mathrm{min}\right)$, frequência respiratória (f, rpm), equivalente ventilatório para o $\mathrm{O}_{2}$ e $\mathrm{CO}_{2}\left(\dot{\mathrm{V} E} / \dot{\mathrm{V}}_{2}\right.$ e $\left.\dot{\mathrm{V} E} / \dot{\mathrm{VCO}}_{2}\right)$, pressão expiratória final de $\mathrm{O}_{2}$ e $\mathrm{CO}_{2}$ $\left(\mathrm{P}_{\mathrm{EF}} \mathrm{O}_{2}\right.$ e $\left.\mathrm{P}_{\mathrm{EFCO}}, \mathrm{mmHg}\right)$ e tempo inspiratório, expiratório e total ( $\mathrm{T}_{\mathrm{I}}, \mathrm{T}_{\mathrm{E}}$, e $\mathrm{T}_{\mathrm{TOT}}$, seg). Cada respiração foi individualmente considerada, o que permitiu uma elevada densidade de pontos e um acompanhamento muito próximo das variações rápidas nas frações gasosas expiradas. Eletrocardiograma de 12 derivações foi continuamente monitorado. Os indivíduos foram 
questionados acerca da sensação de esforço ventilatório e cansaço nos membros inferiores a cada minuto, de acordo com a escala categórica de Borg. Além disso, a determinação da saturação da hemoglobina com oxigênio foi realizada com oxímetro de pulso da marca Onyx ${ }^{\circledR}$ (Nonim, Plymouth, MN, USA) durante todo o teste, assim como a aferição da pressão arterial.

A calibração do módulo de análise de gases foi realizada antes de cada teste, utilizando uma amostra gasosa de referência, com $21 \%$ de oxigênio em balanço nitrogenado. A mistura de calibração foi de $12 \%$ de oxigênio e $5 \%$ de gás carbônico, com mistura de balanço nitrogenado. Após a calibração, o paciente foi instruído quanto as etapas do teste, como a elevação contínua da carga (incremental) e a cessação do exercício de acordo com recomendações consensuais. Foi utilizado um período de repouso de 2 minutos, seguido de 2 minutos sem carga, pedalando a $60 \mathrm{rpm}$ e, enfim, carga incremental de 3 a $10 \mathrm{~W} / \mathrm{min}$, selecionada de acordo com a avaliação clínica e objetivando um teste com duração de 8 a 12 minutos.

\section{Analise estatística}

Para a análise estatística do conjunto de dados foi utilizado o programa SPSS 19, com a aplicação do Teste $\mathrm{t}$ Student ou teste de Mann-Whitney para observação de correlações para variáveis contínuas, conforme a natureza da distribuição. A avaliação da distribuição foi realizada por meio dos testes Kolmogorov-Smirnov e Shapiro-Wilk para variáveis contínuas. O nível de significância utilizado foi de $\mathrm{p}<0,05$.

\section{RESULTADOS}

\section{Característica da amostra}

Foram coletados 46 prontuários de adolescentes sedentários diagnosticados com cardiopatia congênita corrigida cirurgicamente no instituto Dante Pazzanese de Cardiologia no período de junho de 2018 à dezembro de 2019, sendo 8 prontuários 
excluídos por não possuírem teste de exercício cardiopulmonar ou apresentarem dados incompletos. Os dados demográficos dos pacientes incluídos neste estudo estão apresentados na tabela 1 .

Tabela 1 - Características demográficas da amostra $(n=38)$.

\begin{tabular}{lc}
\hline Idade, (anos) & $14,2 \pm 3,8$ \\
Sexo Masculino n $(\%)$ & $20(52,6)$ \\
Sexo Feminino n $(\%)$ & $18(47,4)$ \\
Peso $(\mathrm{Kg})$ & $45,1 \pm 15,5$ \\
Altura $(\mathrm{cm})$ & $150,7 \pm 15,8$ \\
FE $(\%)$ & $68 \pm 7,6$
\end{tabular}

\section{Classificação}

Cianogênicas, n $(\%) \quad 22(57,9)$

Acianogênicas, n $(\%) \quad 16(42,1)$

Legenda: Os dados são expressos em média \pm desvio padrão ou número e porcentagem. Kilogramas $(\mathrm{Kg})$, centímetros $(\mathrm{cm})$, número $(\mathrm{n})$.

Os adolescentes foram avaliados em um único grupo abrangendo as patologias cianogênicas e acioanogênicas. Dentre as cardiopatias 22 (57,9\%) eram cianogênicas e $16(42,1 \%)$ acianogênicas. Os tipos mais frequentes de cardiopatia congênita foram comunicação interventricular (CIV) 12 (31,6\%), comunicação interatrial (CIA) 10 $(26,3 \%)$ e tetralogia de Fallot (T4F) $10(26,3 \%)$.

\section{Teste de exercício cardiopulmonar}


Os dados obtidos antes e após o teste de exercício cardiopulmonar são apresentados na tabela 2 .

Tabela 2. Variáveis no repouso e pico do teste de exercício cardiopulmonar incremental.

Repouso Pico p

\section{Respostas Ventilatórias}

$\mathrm{P}_{\mathrm{ETCO}}$

$\Delta \mathrm{V}_{\mathrm{E}} / \Delta \mathrm{VCO}_{2}$
$33,8 \pm 5,3$

$38 \pm 6,6$
$34,4 \pm 9$

$38,1 \pm 12,7$

\section{Respostas Metabólicas}

$\mathrm{VO}_{2} \mathrm{~L} / \mathrm{min}$

$202,8 \pm 77,4$

$1441,9 \pm 668,8$

0,000

$\mathrm{VO}_{2} \mathrm{ml} / \mathrm{Kg} / \mathrm{min}$

$$
5,1 \pm 2
$$

$33,1 \pm 11,4$

0,000

$\mathrm{VCO}_{2} \mathrm{~L} / \mathrm{min}$

$$
184 \pm 74,9
$$

$1498,6 \pm 719,9$

0,000

RER

$0,89 \pm 0,07$

$1 \pm 0,08$

0,000

\section{Respostas Cardiovasculares}

FC (bpm)

$\mathrm{VO}_{2} / \mathrm{FC}(\mathrm{ml} / \mathrm{min})$

$$
86 \pm 16
$$$$
2,4 \pm 1
$$

$165 \pm 22$

0,000

$8,3 \pm 3,4$

0,000

Legenda: Os dados são expressos em média \pm desvio padrão. $\mathrm{P}_{\mathrm{ET}} \mathrm{CO}_{2}$ : pressão expiratória final de $\mathrm{CO}_{2} ; \Delta \mathrm{V}_{\mathrm{E}} / \Delta \mathrm{VCO}_{2}$ : equivalente ventilatório de dióxido de carbono; $\mathrm{VO}_{2}$ : consumo pulmonar de oxigênio; $\mathrm{VCO}_{2}$ : eliminação pulmonar de $\mathrm{CO}_{2}$; RER: coeficiente de troca gasosa; FC: frequência cardíaca; $\mathrm{VO}_{2} / \mathrm{FC}$ : pulso de oxigênio.

\section{Respostas ventilatórias}

$\mathrm{P}_{\mathrm{ETCO}} \mathrm{CO}_{2}$ não apresentou diferença no pico do esforço não sendo possível indicar se a presença da cardiopatia congênita influenciou a pressão expirada de $\mathrm{CO}_{2}$.

\section{Respostas metabólicas}


Quando analisado o $\mathrm{VO}_{2} \mathrm{ml} / \mathrm{Kg} / \mathrm{min}$ separadamente nas cardiopatias cianogênicas e acianogênicas, foi obtido o valor de 30,6 $\pm 11,2$ e 36,4 $\pm 11,2$ respectivamente, com $p=$ 0,89 .

$\mathrm{O} \mathrm{VO}_{2} \mathrm{~L} / \mathrm{min}$ previsto no pico do TECP foi de $76 \pm 24,5 \%$, quando analisado separadamente cardiopatia cianogênica $(72 \pm 26,8)$ e acianogênica $(81 \pm 20,8)$, não houve diferença entre os grupos $(\mathrm{p}=0,45)$.

O teste foi considerado máximo em 63\% ( $n=24)$ dos adolescentes, pois apresentaram RER > 1 .

\section{Respostas cardiovasculares}

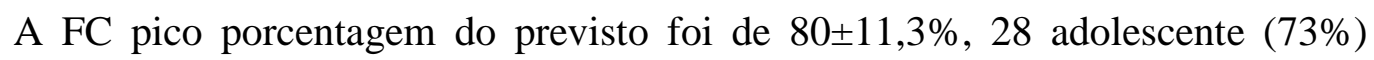
atingiram FC prevista > 70\% demonstrando que apenas 10 adolescentes (27\%) não realizaram um esforço máximo.

O pulso de oxigênio apresentou um incremento esperado após o TECP, quando analisado separadamente as cardiopatias cianogênicas $(8,2 \pm 3,4)$ e acianogênicas $(9,3 \pm 3,7)$ não houve diferença entre as patologias $(p=0,75)$.

\section{DISCUSSÃO}

A OMS afirmou que a capacidade funcional reflete melhor o impacto de uma doença crônica na qualidade de vida. De fato, em adultos com insuficiência cardíaca crônica, a captação máxima de oxigênio $\left(\mathrm{VO}_{2}\right.$ máx $)$ se correlaciona com a qualidade de vida e com o prognóstico, porém tais recomendações sobre o TECP não existem na cardiologia pediátrica, embora mais e mais cardiologistas pediátricos prescrevam o TECP no acompanhamento regular de seus pacientes ${ }^{14}$.

O teste de exercício cardiopulmonar (TECP) tornou-se o 'padrão ouro' para quantificar a gravidade da doença, pois a captação máxima de oxigênio $\left(\mathrm{VO}_{2}\right.$ máx $)$ se correlaciona com a qualidade de vida, prognóstico, capacidade funcional, diagnóstico, quantificação de sintomas e avaliação da resposta ao tratamento ${ }^{15}$. Porém os valores para crianças e adolescentes podem fornecer informações diferentes das obtidas em adultos devido a diferenças nas respostas fisiológicas e metabólicas ao estresse.

$\mathrm{O} \Delta \mathrm{V}_{\mathrm{E}} / \Delta \mathrm{VCO}_{2}$ é a razão entre a taxa ventilatória instantânea e a taxa de liberação de $\mathrm{CO}_{2}$, sendo utilizado como indicador de eficiência ventilatória, ou seja, a resposta 
ventilatória aos estímulos metabólicos é um marcador prognóstico e de gravidade em cardiopatas adultos quando maior que 27 em homens adultos sedentários e maior que 28 em mulheres adultas sedentárias ${ }^{15}$. Na nossa amostra o $\Delta \mathrm{V}_{\mathrm{E}} / \Delta \mathrm{VCO}_{2}$ foi $38,1 \pm 12,7$ podendo sugerir uma ineficiência ventilatória nessa população, porém não há valor de corte para o grupo estudado.

Na nossa amostra o $\mathrm{P}_{\mathrm{ETCO}} \mathrm{CO}_{2}$ apresentou um comportamento normal, ele reflete o $\mathrm{CO}_{2}$ capilar e constituem-se nos valores de pressão parcial do gás exalado na porção final da expiração podendo representar razoavelmente as pressões alveolares médias de $\mathrm{CO}_{2}$, sendo utilizadas para verificar o limiar de lactato $^{15}$.

Schaan et al. em seu estudo obteve o valor de $\mathrm{VO}_{2} 9,31 \mathrm{ml} / \mathrm{kg} / \mathrm{min}$ em adolescentes com cardiopatia congênita cianogênica e acianogênica não corrigida cirurgicamente. No nosso estudo os adolescentes eram corrigidos cirurgicamente e apresentaram o valor superior $33,1 \pm 11,4 \mathrm{ml} / \mathrm{Kg}$, demonstrando uma melhor capacidade funcional.

De acordo com a classificação de Rodrigues et al. ${ }^{17}$ os dados obtidos do $\mathrm{VO}_{2}$ $\mathrm{ml} / \mathrm{Kg} / \mathrm{min}$ na nossa amostra mostram que os adolescentes possuem uma aptidão cardiorrespiratório fraca $(<33 \mathrm{ml} / \mathrm{Kg} / \mathrm{min})$. Quando analisado separadamente as cardiopatias cianogênicas e acianogênicas foi observado que os pacientes com cardiopatia cianogênica apreserentaram aptidão cardiorrespiratória muito fraca e nas cardiopatias acianogênica aptidão fraca, porém não houve significância entre os grupos $(\mathrm{p}=0,89)$. Esses resultados demonstram a necessidade e a importância de estimular a prática de atividades físicas em crianças $\mathrm{e}$ adolescentes, prevenindo $\mathrm{o}$ agravamento $\mathrm{e}$ proporcionando melhor aptidão física.

Apesar dos efeitos positivos da cirurgia, verificou-se que a baixa capacidade de exercício é um preditor independente de morte ou hospitalização, a não realização de atividade física ou o sedentarismo resultam em limitações cardiopulmonares, musculares e psicossociais. Os avanços cirúrgicos aumentam dramaticamente as taxas de sobrevivência de crianças e adolescentes com doença cardíaca congênita. ${ }^{18}$

A resistência periférica total em crianças é mais elevada devido a menor massa muscular e menor tamanho do coração que resultam em menor retorno venoso e acabam contribuindo para um menor volume sistólico, tornando o aumento da frequência cardíaca 
o mecanismo compensatório mais importante para aumentar o débito cardíaco ${ }^{19}$. Na nossa amostra as crianças atingiram FC pico de $165 \pm 22$ bpm (80\% da frequência cardíaca máxima prevista). A frequência cardíaca máxima, a reserva cardíaca e o índice cronotrópico são menores em crianças jovens com cardiopatia congênita ${ }^{19}$. Por esse motivo era esperado que as crianças jovens incluídas neste estudo não conseguissem realizar um teste de esforço máximo, porém $73 \%$ conseguiram atingir valores maiores que $70 \%$ da frequência cardíaca.

Pulso de oxigênio consiste no volume de oxigênio extraído a cada batimento cardíaco e depende do volume de sangue oferecido e da avidez tissular em captá-lo. No nosso estudo o pulso de $\mathrm{O}_{2}$ apresentou incremento significativo após o TECP, embora não seja possível verificar se os valores atingiram o esperado por não haver valores de referência para adolescentes. Em adultos o valor esperado era $>12 \mathrm{ml} / \mathrm{min}$ em homens sedentários e $>10 \mathrm{ml} / \mathrm{min}$ em mulheres sedentárias ${ }^{15}$.

\section{CONCLUSÃO}

Foi possível concluir através desse estudo que os adolescentes em pós-operatório de correção de cardiopatias congênitas cianogênica e acianogênica apresentam aptidão cardiorrespiratória fraca. 


\section{REFERÊNCIAS}

1. Pfeiffer MET et al. Hipertensão Arterial Pulmonar: Abordagem Clínica, Diagnóstica e Avaliação Funcional. 2014; 20(2):50-54.

2. Binder RK, et al. Methodological approach tothe first and second lactate threshold in incrementalcardiopulmonary exercise testing. Eur J Cardiovasc Prev Rehabil. 2008; 15:726-734.

3. Colmenero JC, et al. Hipertensión pulmonar associada a cardiopatias congénitas y síndrome de Eisenmenger. 2015; 85(1):32 - 49.

4. Costa $\mathrm{CH}$, Rufino R. Pulmonary Arterial Hypertension associated to congenic heart disease.

5. Guimarães GV, et al. VO2 Pico e Inclinação VE/VCO2 na Era dos Betabloqueadores na Insuficiência Cardíaca: uma Experiência Brasileira. 2007; 88(6): 624-628.

6. Sousa A, et al. Oxygen uptake kinetics at moderate and extreme swimming intensities. $2013 ; 19$.

7. Moola F, et al. Physical activity participation in youth with surgically corrected congenital heart disease: devising guidelines so Johnny can participate. Paediatr Child Health Mar. 2009; 14(3): 167-70.

8. Budts W. et al. Physical activity in adolescents and adults with congenital heart defects: prescription of individualized exercises. 2013; 34: 3669-3674.

9. Kuang HY, et al. The effectiveness of bosentan endothelin receptor antagonist for pulmonary arterial hypertension associated with congenital heart disease: A systematic review and meta-analysis. 2018; 97 (10).

10. Pelliccia A, et al. Recommendations for competitive sports participation in athletes with cardiovascular disease: a consensus document from the Study Group of Sports Cardiologyof theWorking Group of Cardiac Rehabilitation and Exercise Physiology andtheWorking Group of Myocardial and Pericardial Diseases of the European Societyof Cardiology. Eur Heart J. 2005; 26:1422-1445. 
11. Stoudemire NM, et al. The validity of regulating blood lactate concentration during running byratings of perceivedexertion. MedSci Sports Exerc.1996; 28: 490.

12. Takken $\mathrm{T}$, et al. Recommendations for physical activity, recreation sport, and exercise training in paediatric patients with congenital heart disease: a report from the Exercise, Basic \& Translational Research Section of the European Association of Cardiovascular Prevention and Rehabilitation, the European Congenital Heart and Lung Exercise Group, and the Association for European Paediatric Cardiology. Eur J Cardiovasc Prev Rehabil Aug 222011.

13. Werner Budts, et al. Physical activity in adolescents and adults with congenital heart defects: individualized exercise prescription 2013; 34, 3669-3674.

14. Amedro P, et al. Cardiopulmonary fitness in children with congenital heart diseases versus healthy children. 2017; 312-339.

15. Neder J. A, Nery L. E. Teste de exercício cardiopulmonar. 2002; 28(3): 166-205.

16. Schaan C. W, et al. Functional Capacity in Congenital Heart Disease: A Systematic Review and Meta-Analysis. 2017; 109(4):357-367.

17. Rodrigues A. N., et al. Maximum oxygen uptake in adolescents as measured by cardiopulmonary exercise testing: a classification proposal. 2006; 82(6): 426-430.

18. Neto M. G., et al. Impact of Exercise Training in Aerobic Capacity and Pulmonary Function in Children and Adolescents After Congenital Heart Disease Surgery: A Systematic Review with Meta-analysis. 2015;

19. Scheidt F. V et al. Heart Rate Response During Treadmill Exercise Test in Children and Adolescents With Congenital Heart Disease. 2019; 65 (7). 